\title{
Articles intrépides : le journalisme hybride d'Hervé Guibert
}

\author{
Stéphane Roussel
}

Contrairement à une certaine tradition qui voudrait que l'objectivité soit apparue dans le reportage lors de la guerre de Sécession aux États-Unis et répandue ensuite de par le monde, le journalisme a, semble-t-il, toujours balancé entre les partisans d'une écriture «fleurie »1 et les partisans d'un strict relevé des faits (Ruellan, 2007, p. 126).

\footnotetext{
1 Ruellan (p. 95) cite Théophraste Renaudot, qui, dès 1631, dans sa Gazette, s'interroge sur le style à adopter pour rapporter les événements : «Il s'en trouve qui ne prisent qu'un langage fleuri, d'autres qui veulent que mes relations ressemblent à un squelette décharné, de sorte que la relation en sort toute nue.»
} 
Selon les époques, l'une ou l'autre tendance a variablement prédominé. Dans les années 1960, une tendance apparue aux États-Unis, le New Journalism, a remis au goût du jour le journalisme littéraire, en pratiquant des techniques propres à l'écriture romanesque, déjà anciennes, comme le flash-back, la retranscription intégrale des dialogues, la subjectivité du narrateur ou en abordant des sujets comme la sexualité ou la drogue (voir Le Bohec, p. 411; Demougin, p. 808).

Dans les années 1970, en France, la presse, notamment Libération, semble touchée par ce phénomène (Ruellan, p. 147). De son côté, Hervé Guibert, dans Le Monde, entre 1977 et 1985, écrira aussi des articles influencés par la littérature. Les procédés qu'il utilise sont bien ceux que l'on repère dans le New Journalism, mais il en apporte d'autres, qui lui sont personnels : la multiplication des «pistes » narratives, le contraste entre les thèmes, innocents ou crus $^{2}$, entre les styles, neutre ou sophistiqué, entre la présence ou l'absence du narrateur, entre traitement de l'information référentielle et jeux multiples sur le signifiant.

Ainsi, l'expérience journalistique de Guibert va au-delà d'une simple réutilisation de procédés littéraires préexistants. Il écrit ses articles comme il écrit son œuvre, en repoussant toujours les limites d'ordre générique, comme celles qui, dans le journalisme, interdit le recours à la première personne ou à la fiction. En cela, il s'inscrit dans une tendance littéraire qui témoigne d'un "éclatement des genres », repérable chez les plus grands auteurs du XXe siècle (Dambre et Gosselin-Noat). C'est par le biais original du journalisme mis en relation avec la

\footnotetext{
${ }^{2}$ Guibert confie dans son journal intime qu'il a écrit une œuvre « délicate et barbare » (Guibert, 2001).
} 
littérature, problématique généralement peu étudiée (Boucharenc, p. 12), que nous allons interroger ce phénomène, en particulier dans Articles intrépides, paru en 2008.

\section{Les écarts narratifs}

Guibert s'intéresse dans la narration à tout ce qui vient complexifier l'ordre, l'enchaînement narratif. Cette tendance peut conduire à une digression totale par rapport au sujet annoncé. Pour reprendre une expression de l'auteur, on peut dire que sa conception du journalisme se définit comme une « dérive » des « regards » $(A I$, p. 330).

En parlant d'un film qui use de ce procédé comme Empty quarter de Raymond Depardon, Guibert favorise alors une interaction entre l'écriture filmique et l'écriture journalistique. Par un double procédé de mise en abyme et de retour en arrière, il rapporte la discussion qu'il a eue avec Depardon à la sortie d'un autre film, L'État des choses de Wim Wenders, au Festival de Venise, parce que cette conversation entre en résonance avec le thème même du film de Depardon, à savoir une digression narrative :

Finalement, nous nous en fichons de parler bien ou mal de ce film, de dire exactement comment nous l'avons aimé ou moins aimé : la nuit tombe lentement, des corps passent devant nous, à pied, à bicyclette, solitaires ou en bandes, rieurs ou mystérieux, et nos yeux sont accrochés différemment, ce sont ces silhouettes pâles ou bronzées qui les mènent, qui les assujettissent et en règlent le mouvement, il y a comme ça un on. [...] (l'idée de départ d'Empty Quarter est exactement ça : envoyé comme reporter en pleine guerre du Vietnam, Depardon se met à ne plus rien voir que le corps d'une femme qui est entrée par hasard dans son hôtel, puis dans sa chambre, puis dans sa vie, et rétrécit son champ de vision sur ses jambes 
nues par exemple, ou sur sa nuque de garçonne, et cette obnubilation repousse tout hors du champ : la guerre, l'histoire, la morale, la raison professionnelle). (AI, p. 329-330)

On peut aisément appliquer ce procédé cinématographique à l'écriture journalistique de Guibert. Pour enquêter sur le grand spectacle parisien, il s'immisce dans les coulisses et nous donne à voir l'envers du décor. Plutôt que de faire une analyse du fonctionnement du grand spectacle en suivant un plan logique et rationnel, ou de poser des questions, d'émettre des jugements, de hiérarchiser les données, Guibert "enregistre », sans trame narrative, dans le désordre, les mouvements, les gestes, les paroles, les infinis détails des différents acteurs qui contribuent à la création du spectacle et qui donnent cette impression d'enchaînement virevoltant, de vitesse troublante et enivrante, due à la « giration de ces corps » que le regard fait défiler :

Le régisseur fait une annonce au micro : «Les artistes du corps de ballet et du chœur désireux de toucher un acompte sont priés de se rendre au bureau de M. Untel. » Le hussard est affalé sur un ancien décor : " Mais qu'est-ce que tu fais là ? - Je suis fatigué, la fièvre me fout par terre. » L'habilleuse, qui ne manque pas un tableau, suit le spectacle par un rideau du côté et bat la mesure en même temps que le chef d'orchestre. ( $A I$, p. 52)

Cet enregistrement du réel peut être rapproché de la retranscription totale des paroles des personnes interrogées lors des entretiens. Selon Bruno Blanckeman, cette tendance est liée à l'époque :

Il se laisse aussi influencer par un phénomène d'époque, l'influence de médias qui diffusent une langue à la fois plus souple et moins complexe, compréhensible dans l'instant de son émission; la vogue des témoignages enregistrés puis retranscrits. (2008, p. 129)

Le défilé des corps, dans cette écriture très cinématographique, peut aussi suspendre et mettre en valeur un geste, révélateur 
d'une émotion ou d'un sentiment. Cet instantané est sans doute plus révélateur que tout autre procédé qui n'aurait peut-être pas été à même de capter l'expression spontanée de ce sentiment, la beauté d'une pure émotion. On peut rapprocher ce procédé du cinéma, mais d'un cinéma exempt du déroulement narratif traditionnel, comme celui que représente à merveille pour Guibert le Loulou de Pabst, a contrario des films plus récents :

[...] le cinéma s'est affaibli à l'arrivée du parlant, en se calquant sur une forme romanesque littérale et en perdant son caractère photographique. (AI, p. 105)

Faire un récit amène inévitablement à modifier la réalité, par abus de simplification, et conduit à émousser l'émotion du spectateur ou du lecteur. Là où la vie réelle est désordre, mélange de pensées, d'actions, de rêves, de fantasmes, l'œuvre d'art doit rendre compte de ce désordre.

La dynamique qui se substitue à la trame narrative et romanesque se recrée aussi dans le défilement des décors. Elle fait écho à un procédé cinématographique que Guibert a repéré dans Le Voleur de bicyclette de Vittorio de Sica :

À l'enchaînement des éléments du scénario, comme un exercice de fatalité, succède l'enchaînement des décors, comme un exercice de diversion : mont-de-piété, église, bordel, trattoria... (AI, p. 275).

À Barcelone, il emprunte le dédale des rues tortueuses des ramblas où on lui propose de la drogue, puis il erre dans les night-clubs (le Molino, la villa Roja, la Bodega Bohemia, la Paloma), dans une fête foraine au petit matin, un musée de cire, une cathédrale, un camion projetant « des photos de guerre et d'enfants décharnés » (AI, p. 96), sur la plaza Catalunya pour 
terminer au moment où le soir descend et avec la vision d'un enfant qui tue un oiseau. L'enchaînement des lieux est troublant et mélange les registres comme chez Vittorio de Sica : l'innocence et la perversité, le sacré et le profane, jusque dans la cathédrale où « une vieille prostituée » et de «vieilles femmes dignes » se côtoient.

Chaque lieu traversé est un morceau du puzzle de la capitale catalane et les rues traversées forment un labyrinthe où le journaliste cherche à s'égarer, comme dans Citizen Kane d'Orson Welles. Le début de l'article où grouillent le vice et la puanteur, les gens louches et les propositions interlopes, les voyous et les prostitués, rappelle les univers de Genet, de Fassbinder ou de Pasolini. Il entre en dissonance, après un long périple et des détours multiples et hétéroclites, avec la fin de l'article, qui décrit deux enfants insouciants et libres chassant des oiseaux pour les vendre à des empailleurs : une scène de rue qui aurait pu être photographiée par Robert Doisneau, photographe de la rue, ou filmée par François Truffaut, cinéaste de l'enfance. Le contraste livre une quintessence à cette scène finale et la chute distille une émotion rare, sans être dans la certitude, pour la première fois repérée et découverte in extremis au hasard de la déambulation du journaliste : « Il a l'air heureux. » (AI, p. 97)

Ce procédé narratif semble être ici un exemple simple de ce qui deviendra un principe d'écriture dans les « récits de l'âge du mal», selon l'expression de Bruno Blanckeman. Celui-ci écrit, à propos de À l'ami qui ne m'a pas sauvé la vie :

Le récit s'épuise en considérations annexes, en détails excroissants, comme s'il voulait capter les rebuts du quotidien, les réflexes prosaïques, cette part perdue de la vie dont la gratuité concentre justement le principe. Faits, constats, 
impressions, jugements saturent le récit et dissipent sa discipline. Les anecdotes jaillissent sans contrôle : l'absence de sélection, de hiérarchie, parfois de nécessité, suscite l'illusion d'une réalité protéiforme. (p. 125)

L'écriture s'émancipe de son sujet, revendique son autosuffisance esthétique, réclame ses incursions vers l'inattendu, l'étrange, l'insolite.

\section{L'hybridité générique}

Guibert cherche à traiter son sujet de manière détournée, en jouant sur certaines limites liées au genre journalistique, en particulier celle de la fiction et de l'expression de soi.

Ainsi "Passion délétère ", un article consacré à Amanda Lear, tient à la fois de la nouvelle et de l'article. Certains passages relèvent bien en effet du journalisme : on reconnaît le rappel biographique de la chanteuse, son actualité, l'entretien avec questions et réponses. Mais ces passages journalistiques sont insérés dans une trame romanesque. La narration est menée du point de vue d'un personnage fictif : Nicky Mangano, lui aussi journaliste. Il tombe amoureux d'Amanda Lear en entendant sa voix, cherche à la rencontrer, est déçu par un rendez-vous manqué et finit par ourdir une perfide vengeance. La fiction est soulignée par le burlesque des origines du journaliste, l'aventure amoureuse, le fantastique de la boîte qui contient des «reliques» de la chanteuse. Selon Todorov, la fiction est un caractère et un indice essentiels de littérarité.

Le caractère hybride de l'article est lui-même souligné et mis en abyme par la thématique (conférence sur l'hermaphrodisme des papillons mouchetés - l'impureté se 
signale à tous les niveaux - à laquelle le journaliste doit assister), par l'onomastique (le nom du journaliste est la réunion du prénom du père et du nom de la mère, eux-mêmes incarnations et parangons de la masculinité et de la féminité) et par la rumeur liée à la transsexualité d'Amanda Lear.

L'hybridité de cet article est aussi celle qui consiste à appliquer une écriture cinématographique qui brouille encore un peu plus la frontière entre les genres :

Il traînait sa solitude dans la gare, encadré par deux carabiniers qui avaient de ces plumages noirs sur leurs casques, quand une voix rauque sortant tout à coup du juke-box le fascina, elle chantait Queen of China Town. Il chercha sur l'appareil argenté le numéro de la chanson et trouva ces deux mots qui le fascinèrent peut-être plus que la voix : Amanda Lear. Il l'oublia d'abord. Mais quatre mois plus tard, alors que son journal l'avait envoyé à Salzbourg pour assister à une expérimentation du rayon de la mort, et qu'il aspirait dans un milchbar la mousse de son bananen-shake, la voix fatidique se fit de nouveau entendre. Amanda Lear chantait Follow me, Nicky était devenu un fan. (AI, p. 38)

Cet extrait mélange le burlesque («une expérimentation du rayon de la mort»), la cristallisation amoureuse et les références à l'atmosphère des films américains. Les ruptures temporelles évoquent le montage et l'enchaînement en séquences de l'écriture cinématographique.

La limite de ce genre d'articles consiste en une perte des repères du lecteur. Traditionnellement, le lecteur d'un article n'accède pas, contrairement au lecteur de fiction, au célèbre « renoncement consenti à l'incroyance » défini par Coleridge : il est censé adhérer à la réalité de ce que le journaliste exprime. Cela est lié à la convention générique liée à l'article de journal. Finalement, même si certains éléments sont vrais, l'entourage 
de fiction dans lequel ils s'insèrent met en doute la véracité de l'ensemble des informations.

$\mathrm{Au}$ terme de «reportage », Guibert préfère le mot " enquête", qu'il emploie souvent dans les sous-titres de ses articles. Ce mot a l'avantage de mêler les sèmes policier et journalistique, brouillant les frontières génériques entre écriture journalistique et roman noir ou nouvelle policière, substituant le journaliste à l'enquêteur toujours prompt à dévoiler quelques mystères, quelques secrets. Blanckeman note à cet égard :

La fonction romanesque enquêteur/détective/reporter, récurrente dans l'œuvre, devient emblématique de cette traque d'un autre auquel peu à peu le narrateur s'identifie, selon un jeu de change identitaire qui rappelle certains récits de Paul Auster. (2008, p. 123)

Ainsi, une enquête à l'Armée de salut amène Guibert à se faire passer pour un vendeur de chaussures, puis à endosser l'uniforme d'un militaire pour intégrer une chorale. Guibert se dédouble, multiplie les identités : journaliste, faux vendeur, faux militaire. Là encore, la réalité et la fiction, la vie et l'art se mêlent indissociablement: une scène du Loulou de Pabst, cité dans l'article, se passe justement à l'Armée du salut, le soir de Noël où Jack l'Éventreur a tué Loulou. Guibert sera-t-il la victime ou le bourreau ? Il décrit alors une atmosphère de fauxsemblant, apparemment lourde de menaces, de suspicions, proliférant dans l'écriture de l'article. Le «moi » tend alors à se fictionnaliser, devient personnage, et fictionnalise la réalité observée, amorce des possibilités d'intrigue policière :

La première visite, incognito, recèle déjà une grande étrangeté : sur le trottoir d'en face, un homme essaie une paire de jumelles, un autre homme complètement tatoué fait irruption parmi les 
vierges strictes, le $\mathrm{S}$ cousu au fil d'or sur l'épaulette de l'uniforme bleu marine, cet homme porte une caisse de spiritueux, figurez-vous. (AI, p. 149)

Après avoir craint d'être pris au piège, Guibert se voit bien prendre la place de Jack l'Éventreur pour se venger de la remarque perfide sur ses piètres qualités de chanteur. Puis le récit, qui suit cette fois une ligne narrative, part dans une autre direction et glisse vers le burlesque, désamorce la piste policière: les personnes, d'abord inquiétantes, apparaissent désormais gentiment loufoques.

L'article ne cesse de jouer sur la "rupture de lignes », complexifiant la trame narrative qui est très simple et visant même à la faire oublier, à se substituer à elle, à l'instar d'un cinéma qui plaît à Guibert : réalité ou fiction, dialogues ou récit, observation ou action, policier ou burlesque. Les modes de représentation sont inversés : la réalité paraît suspecte, le faux a un caractère de vérité : "Ici les barbes ont l'air postiche, les bosses aussi, seul le simili-léopard des cols a un air de vrai » ( $A I$, p. 152). Les références aux œuvres de fiction Loulou de Pabst et Alice de Lewis Carroll rappellent la tentation de basculer de l'autre côté du miroir du réel, de retrouver dans la réalité les sensations éprouvées au cinéma et qui n'est peut-être que l'expression du « désir fou » de Guibert pour le cinéma. Ce désir s'origine en tout cas et explicitement dans la volonté de ressentir les mêmes sensations qu'un «cœur simple», rappelant la Félicité de Flaubert :

Mais comment donc faire partie de ces cœurs simples, si joliment habillés et qui agitent leurs clochettes en regardant tomber les billets de banque, comment se joindre au cortège choral, comment connaître le plaisir de la gerçure sous mitaine, du gel sous les bottillons fourrées? (AI, p. 150) 
L'écriture porte donc, à travers la mise en danger fantasmée de soi, la trace du principe de piratage du réel, du désir de s'en émanciper, quitte à en oublier le sujet de l'article. Comme l'explique Blanckeman :

Écriture et sujet se poussent à bout. La première exprime ce qui d'une existence pourrait être, le second extériorise ce qui agit à la lisière de sa conscience. La démarche de Guibert est moins psychologique qu'exologique - se chercher hors de soi, dans une fuite en avant expérimentale - et infralogique - solliciter son identité virtuelle. (2008, p. 124-125)

Dans "Parcours ensorcelé », Guibert s'amuse également à perdre son lecteur dans les méandres du vrai et du faux. L'article se présente sous la forme de 24 propositions que le lecteur doit déterminer comme vraies ou fausses. Les réponses sont données en fin d'article, écrites à l'envers et en petits caractères, comme dans les jeux proposés en fin de journaux. La forme de l'article coïncide parfaitement avec son sujet puisqu'il rend compte d'une exposition sur le vivant et l'artificiel au Festival d'Avignon. Mais le lecteur est vite perdu, car certaines propositions sont déclarées vraies en dehors de tout rationalisme :

Emmanuel Ostrovski, qui proposera un parcours théâtral nocturne dans les chambres du deuxième étage (consulter la liste des manifestations), s'élèvera, sans l'aide d'aucun levier ni d'aucune supercherie, de 5 centimètres au-dessus des toits, le 12 juillet à 23 heures pile, pour démontrer son caractère angélique. (AI, p. 307)

Inversement, des vérités évidentes sont annoncées comme fausses, à la faveur de l'ambiguïté de la référence du pronom «vous». La proposition est vraie si le «vous» renvoie à l'exposition "vivant/artificiel » placé en apposition en tête de phrase ; elle est fausse si le « vous » renvoie au lecteur : 
Vivant/artificiel: vous êtes vous-mêmes, n'est-ce pas, on ne peut plus vivant (si vous doutez, pincez-vous, et continuez le parcours). (AI, p. 307)

Guibert, après avoir donné les réponses, précise, non sans humour :

La plupart de ces réponses étant, pour des raisons qui tiennent au secret professionnel, erronées ou apocryphes, il est conseillé de se reporter aux réponses exactes qui seront publiées dans le journal Le Monde du 11 juillet 1994, en vente par souscription. (AI, p. 309)

Il énonce ainsi une vérité, mais clôt aussitôt sur un autre mensonge, renvoyant le lecteur à l'indétermination de toute réponse permettant de statuer définitivement. On retrouve ici un procédé de manipulation des données du réel que Guibert utilise dans son œuvre romanesque pour appréhender le fonctionnement du psychisme. Comme le souligne Blanckeman, pour le romancier, le «faux n'est pas l'ennemi du vrai, mais sa perspective tournante », car " tout sens unilatéral, du sentiment comme du récit, en fausse la vérité. » (2008, p. 110)

Guibert désire faire naître chez son lecteur la même trouble sensation de pertes de repères que celle qu'éprouveront les spectateurs. Le caractère fictif sur lequel jouent cette exposition et ce spectacle rejaillit sur l'écriture de l'article, comme si la forme de l'article devait être contaminée par le fond.

Un entretien entre plusieurs comédiens est propice à une mise en forme ad hoc du texte. Typographiquement, le texte emprunte à la fiction. Chaque comédien voit ses paroles introduites par l'indication de son nom en capitales suivi d'un tiret, comme dans un dialogue de théâtre ou de cinéma. Guibert intervient de temps à autre pour relancer la discussion. Mais là 
encore, ses paroles sont habilement indiquées en italique et entre parenthèses, comme s'il s'agissait de didascalies auctoriales. Le mimétisme est alors total entre l'article journalistique et le texte de fiction. C'est aussi le cas dans un entretien avec Patrice Chéreau à propos de sa mise en scène de Lulu d'Alan Berg. L'article se présente sous l'aspect d'une chronique de sa création, une sorte de journal de bord, en indiquant en italique avant chaque paragraphe le mois dont il est question, du début du projet à sa réalisation effective. Il donne ainsi une dynamique narrative à l'article, retraçant un historique de la genèse du spectacle, créant une sorte de suspense (le voyage en Allemagne de Chéreau pour rencontrer Boulez, une grève des machinistes, la fin de la grève et le bouclage des répétitions in extremis) que la seule retranscription des paroles de Chéreau n'aurait pas aussi bien mis en valeur.

Nouvelle, questionnaire ludique, dialogue, journal de création, l'article emprunte de nombreuses formes originales et inattendues, souvent littéraires.

Une autre contrainte liée au genre journalistique est celle qui interdit de parler de soi. Or, de nombreux articles s'émancipent de ce principe et témoignent d'une hésitation entre le récit-document et le récit-monument, pour reprendre des termes employés par Blanckeman.

Certains incipits ou excipits d'articles sont ainsi écrits à la première personne; comme si l'évacuation du «Je» était impossible. C'est le cas par exemple de la fin de la longue enquête sur Balthus, qui se termine par une remarque très personnelle : 
Mais quelle idée aussi de demander un article sur Balthus à quelqu'un qui est irrémédiablement allergique aux chats et qui, de surcroît, a une peur bleue des petites filles depuis que l'une d'elles — anglaise, comme par hasard —, un soir de 1970, à Seaford, s'est relevée de la cheminée, où elle jouait devant les flammes, pour lui piquer la joue avec une aiguille... (AI, p. 279-280)

D'autres articles s'émancipent totalement de l'actualité, comme «Les désarrois d'un novice», "Too much» ou "J'ai peur de repartir ». Ce sont des textes qui témoignent de l'opinion du moi de l'écrivain sur un sujet particulier: les discothèques, l'Amérique, les voyages. Ils semblent ainsi davantage relever du billet d'humeur, mais pourraient tout aussi bien être extraits d'une page de journal intime ou d'autobiographie.

« Les désarrois d'un novice » est un article composé d'une seule et très longue phrase qui se partage entre des paroles rapportées directement où s'exprime un « Je » en proie à une situation bien réelle, une soirée en discothèque, et l'intervention d'un narrateur qui juge ce «Je». L'écrivain renouvelle ici le projet rousseauiste de Rousseau, juge de JeanJacques; il devient lui-même l'objet de sa propre appréciation. Blanckeman précise à cet égard :

Par ailleurs, l'écriture de l'intimité entretient l'intimité de l'écriture. L'observation de soi impose une pratique textuelle en miroir: quand l'être se regarde, l'écrivain s'appréhende. Le mesure de soi conjoint repères réels et livresques. Si la dimension journalistique du récit met en question son appartenance littéraire, une tension intertextuelle permanente la met en perspective. Hervé Guibert établit son œuvre à leur croisée. (2008, p. 136)

Il semble que cet article illustre ce croisement entre « celui qui la nuit préfère les noircissures des siècles passés plutôt que de faire la fête jusqu'au petit-matin », l'amoureux de la littérature, 
et un « Je» qui s'expérimente à l'extérieur au contact des autres: "il suffirait que je fixe un de ces individus quelques temps et, enfin, je danserai, et je me fondrai anonyme dans la foule » (AI, p. 82). Ce regard spéculaire sur soi ne s'épargne pas et l'autodérision désamorce toute velléité de narcissisme : ainsi n'est-il que «le danseur mesquin et économe, le rabat-joie, le gagne-petit, le complexé » (AI, p. 82-83).

Échapper à soi-même, adopter d'autres points de vue sur le monde que le sien propre, par projection identitaire, permet à Guibert de s'appréhender en l'autre. Le journalisme d'investigation permet aussi d'atteindre l'altérité dans les métamorphoses qu'il autorise. Le «moi » fait l'expérience de l'autre à travers les enquêtes menées et dépasse les limites de l'individualité et de l'identité.

Dans "Rendez-vous avec Vincent», Guibert adopte un point de vue original sur les tableaux de Van Gogh, celui d'une jeune femme qui semble vivre intensément les peintures du peintre hollandais :

Au musée Van Gogh, à Amsterdam, une femme regarde les tableaux. Elle est jeune, petite, très maigre, mais sa maigreur est cachée sous ses bras croisés, son cou disparaît sous un nœud d'écharpe, et ses poignets dans ses manches. Elle semble n'avoir aucun poids, comme si son corps ne pouvait prétendre à aucune possession de l'espace. C'est presque un fantôme. (AI, p. 160)

Ainsi Guibert raconte-t-il l'histoire de cette femme, manière détournée de faire la description des tableaux et le compte rendu de cette exposition. Il évite ainsi la description directe et classique qu'il délègue à une personne dont on ne sait pas vraiment si elle existe, et qui semble vouloir se fondre dans le tableau qu'elle observe, comme dans Comment Wang-fô fut sauvé de Yourcenar. Puis il retrouve son point de vue personnel 
et fait, lui aussi, l'expérience d'une fusion entre la peinture et la réalité :

Pour l'instant, il me semble traverser, physiquement, l'œuvre de Caspar Friedrich : une succession d'écrans embrumés, avec d'innombrables géants noirs aux bras dentelés, parfois courbés, et en larmes de givre, gémissant sous leur gigantisme. ( $A I$, p. 164)

Les entretiens qui laissent souvent totalement la place à la voix de la personne interrogée, outre qu'ils sont un des procédés revendiqués par le New Journalism, apparaissent comme la radicalisation de ce point de vue d'autrui. La part accordée au «Je » est alors réduite au minimum, dans une brève introduction à l'entretien.

Le contraste est alors saisissant entre les articles où le « Je » est omniprésent et les articles où celui-ci est quasiment absent. Il marque la volonté de l'écrivain de multiplier les regards et les voix dans son écriture, créant un effet de polyphonie et de morcellement identitaire.

Les entretiens qui se présentent sous une forme plus classique de questions/réponses laissent souvent entrevoir des préoccupations propres à l'écrivain. Lorsqu'il interroge Deleuze à propos d'un ouvrage qu'il a consacré à la peinture, Guibert est directement intéressé puisqu'il a lui-même envisagé d'écrire sur ce thème. Il est autant le destinataire des réponses du philosophe que le lecteur de l'article.

\section{Le mélange des registres}

D'autres lignes de rupture interviennent dans cette écriture à visée métamorphique. L'une d'entre elles consiste à mélanger 
différents registres, styles ou tonalités : le littéraire côtoie le populaire. Ainsi se côtoient Deleuze et Dalida, Étienne Daho et Jean-Luc Godard. La chanson, la philosophie, le cinéma se mêlent, à la manière de Pasolini dans Théorème.

En citant une longue phrase issue d'un ouvrage du XIX siècle sur les aveugles, Guibert donne à voir un autre type de contraste, celui qui se crée entre une vision passéiste, archaïque, et une vision moderne, actuelle, totalement différente. Il donne à entendre, par ce procédé citationnel, une voix d'outre-tombe et met au jour une archéologie du savoir qui le rapproche de la démarche philosophique de Foucault.

Dans un tout autre registre, le portrait d'Adjani est hautement littéraire, par l'usage de métaphores, de multiples expansions du nom, qui le rapproche d'une certaine préciosité fondée sur la rareté. Le style littéraire, associé à un niveau de langage soutenu, se superpose alors au registre épidictique :

Le corps a de ces métaphores, littéraires et usées, climatiques, minéralogiques. Il faudrait donc me tourner la peau, d'une pâleur et d'une matité d'un autre siècle: une blancheur qui n'est pas poudreuse ni cadavérique mais qui tient de la porcelaine, de la lactescence, et où affleurent si facilement les bouffées roses du trouble, le subtil réseau des veinules, le maquillage inné d'un riche tissu peaucier, un ovale de femme gravé sur un camée ou renfermé précieusement dans le double fond d'un sautoir, une adolescence scandinave. (AI, p. 132)

L'article consacré à la danseuse Pina Bausch débute par une très longue phrase, habituellement bannie du journalisme, comme l'indique Jacques Mouriquand. Le style de Guibert semble se conformer à merveille à l'objet qu'il décrit. Sa phrase est ponctuée de nombreux verbes à l'infinitif, comme pour mieux épouser et traduire les multiples mouvements du 
danseur, auquel est assimilé le lecteur, et se termine par une liste de noms qui donnent l'idée des figures que miment ces mouvements :

Il est arrivé à tout le monde, un jour ou l'autre, d'euphorie spéciale ou de cafard outre mesure, seul ou en société, devant une glace ou dans un coin, sur une musique ou en silence, de faire un pas de travers, de fléchir sa jambe derrière le genou et de laisser balloter ses bras, comme si on allait s'évanouir, et de se reprendre, de devenir un paquet de linge ou une boule de nerfs, pendant quelques secondes, à une gymnastique déglinguée, sans utilité, de dépenser sans but, tracer d'autres gestes dans l'espace, le fouetter, s'appuyer sur lui comme pour $y$ prendre son envol, habiter son corps de postures étrangères, de fièvres lointaines, de réminiscences cinématographiques, devenir la reine de Saba ou Elvis Presley, ou les deux en même temps, les faire lutter, valser en soi au même moment, se mettre dans la peau d'un tigre, d'une Gitane, se parer de cuir ou de falbalas, respirer différemment, souffler, écumer, nager sous l'eau, sirène ou homme-grenouille, oiseau, toupie, gyroscope, pantin, mécanique. (AI, p. 157-158).

On y retrouve l'idée de l'art comme métamorphose de soi. La phrase de Guibert imite donc la danse, comme elle imite le mélange des registres, les ruptures narratives du cinéma.

La préciosité stylistique de certains articles n'efface cependant pas le recours à la crudité pornographique. En consacrant de nombreux articles aux représentations du corps, Guibert fait une large place à "l'image obscène » qui apparaît chez Pasolini au milieu des scènes les plus saintes et les plus nobles, ce qui n'est pas habituel au journalisme, mais qui est une tendance nette de son esthétique, qui se joue de tous les tabous. Les expositions offrent un moyen détourné, celui du récit-document, d'aborder des sujets essentiels pour établir l'œuvre-monument (Blanckeman). 
Le spectacle du corps mis à nu n'est intéressant que dans la mesure où il renvoie à soi-même et incite à une méditation existentielle, réflexion au sens double du terme, tels les memento mori des siècles passés. Ce spectacle spéculaire va audelà d'une simple admiration superficielle; il invite à une introspection sans complaisance, dont l'objectif se concentre toujours sur la connaissance de soi. Mais Guibert ajoute que ce spectacle ne doit pas conduire à une sublimation ou à une édulcoration de la mort dans l'art. Le corps torturé, souffrant, malade, doit attester d'une vérité du moi, en lien direct avec son fonctionnement psychique. L'extrémité de l'exploration du corps et de la recherche de sa vérité se joue de tous les tabous. Guibert aborde tour à tour la nécrophilie ou la zoophilie.

Face à la mort, « on n'a que deux possibilités : l'obsession ou l'oubli»; on peut ajouter que le corps subit le même traitement. Obsession indéniable de Guibert, le corps est touché par la même radicalité du désir de connaissance. L'auteur précise même, non sans humour, à ses lecteurs du Monde, à propos d'une visite au Musée infâme du Docteur Spitzer, où sont exposés dans des bocaux des organes humains, qu'«il ne faut pas jouer les dégoûtés, ni les délicats dans cette histoire, mais les petits enfants vicieux, ou curieux [...]. » (AI, p. 111)

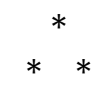

Placés sous le signe des écarts narratifs, de l'hybridité générique et du mélange des registres, les Articles intrépides ne diffèrent finalement pas du reste de l'œuvre de Guibert et de son projet de connaissance de soi. À cet égard, ils rappellent souvent d'autres genres, comme le journal intime, la nouvelle ou l'autobiographie. Le « je » y est omniprésent, ne serait-ce que 
dans le choix des sujets ou du style de l'écriture. Mais aussi, parfois, le «je » s'absente au contact de l'altérité, semblant jouer d'un journalisme aux genres et aux styles protéiformes. Les éléments récoltés par Guibert au cours de ses pérégrinations sont autant de manière d'assouvir sa curiosité et d'approfondir ses choix esthétiques, parallèlement à l'élaboration de son œuvre. Ses premières années de journalisme sont par exemple très marquées par le corps et le morbide; les dernières sont davantage centrées sur le cinéma et la recherche de correspondances possibles avec la littérature; autant de sujets qui sont au cœur de l'œuvre de Guibert.

Les Articles intrépides témoignent aussi d'une tendance du journalisme de cette époque, le New Journalism, qui a certainement pu favoriser leur apparition. Écriture du fragment, du passage rapide d'un sujet à l'autre, propice à capter les petits détails du quotidien, à nous donner à voir et à entendre, l'écriture journalistique a de multiples intérêts qui peuvent servir à forger le style d'un écrivain et à créer un effet « reportage » dans certains passages de l'œuvre. En cela, ils sont aussi le reflet d'une écriture marquée par la remise en question des genres littéraires au XXe siècle. Dès lors, la question : «que fait la littérature au journalisme?» est remplacée par une autre : «que fait le journalisme à la littérature ?» L'œuvre de Guibert mérite d'être relue à l'aune de cette nouvelle question. 


\section{Bibliographie}

BlAnCKEMAN, Bruno. (2008 [2000]), Les Récits indécidables: Jean Echenoz, Hervé Guibert, Pascal Quignard, Lille, Presses universitaires du Septentrion, coll. « Perspectives ».

BOUCHARENC, Myriam. (2004), L'Écrivain-reporter au cour des années trente, Villeneuve d'Ascq, Presses universitaires du Septentrion.

DAmbre, Marc et Monique Gosselin-NoAT. (2001), L'Éclatement des genres au XXe siècle, Paris, Presses Sorbonne nouvelle.

DEMougin, Jacques. (1992), Dictionnaire historique, thématique et technique des littératures, Paris, Larousse.

GuIBERT, Hervé. (1994 [1980]), «Le critique photo », dans La Piqûre d'amour et autres textes, Paris, Gallimard, coll. « Folio ».

—. (2008), Articles intrépides, Paris, Gallimard.

LE BoHec, Jacques. (2010), Dictionnaire du journalisme et des médias, Rennes, Presses universitaires de Rennes.

MouriquAND, Jacques. (1997), L'Écriture journalistique, Paris, P.U.F., coll. «Que sais-je ? ».

RUELLAN, Denis. (2007), Le Journalisme ou le professionnalisme du flou, Grenoble, Presses universitaires de Grenoble.

Todorov, Tzvetan. (1987), La notion de littérature et autres essais, Paris, Seuil, coll. « Points ». 


\title{
Résumé
}

Cet article a pour objectif de montrer que les Articles intrépides ne sont pas le simple commentaire des œuvres d'art dont Hervé Guibert a choisi de rendre compte - peinture, cinéma, danse, etc. -, mais aussi, et surtout, l'expression, par effet de correspondance, de toute la conception esthétique de l'écrivain. En outre, l'article de presse lui-même, dans sa forme, témoigne d'une écriture qui ne connaît aucune frontière générique, justifiant sans doute son caractère intrépide et qui applique au texte les procédés, cinématographique ou photographique mis au jour dans les œuvres de prédilection.

\begin{abstract}
This article aims to show Articles intrépides is not the simple explanation of works of art he has chosen to report - painting, film, dance ... but also and above all the expression, effect correspondence, all the aesthetic design of Hervé Guibert. In addition, the press release itself, in its form, which reflects a write knows no generic, perhaps justifying his fearless nature, and applies to the text processes, film and photography, brought to light in the works of choice.
\end{abstract}

